TRANSACTIONS OF THE

AMERICAN MATHEMATICAL SOCIETY

Volume 352, Number 10, Pages 4469-4486

$\mathrm{S} 0002-9947(00) 02571-\mathrm{X}$

Article electronically published on June 13, 2000

\title{
WILLMORE TWO-SPHERES IN THE FOUR-SPHERE
}

\author{
SEBASTIÁN MONTIEL
}

\begin{abstract}
Genus zero Willmore surfaces immersed in the three-sphere $\mathbb{S}^{3}$ correspond via the stereographic projection to minimal surfaces in Euclidean three-space with finite total curvature and embedded planar ends. The critical values of the Willmore functional are $4 \pi k$, where $k \in \mathbb{N}^{*}$, with $k \neq 2,3,5,7$. When the ambient space is the four-sphere $\mathbb{S}^{4}$, the regular homotopy class of immersions of the two-sphere $\mathbb{S}^{2}$ is determined by the self-intersection number $q \in \mathbb{Z}$; here we shall prove that the possible critical values are $4 \pi(|q|+k+1)$, where $k \in \mathbb{N}$. Moreover, if $k=0$, the corresponding immersion, or its antipodal, is obtained, via the twistor Penrose fibration $\mathbb{P}^{3} \rightarrow \mathbb{S}^{4}$, from a rational curve in $\mathbb{P}^{3}$ and, if $k \neq 0$, via stereographic projection, from a minimal surface in $\mathbb{R}^{4}$ with finite total curvature and embedded planar ends. An immersion lies in both families when the rational curve is contained in some $\mathbb{P}^{2} \subset \mathbb{P}^{3}$ or (equivalently) when the minimal surface of $\mathbb{R}^{4}$ is complex with respect to a suitable complex structure of $\mathbb{R}^{4}$.
\end{abstract}

\section{INTRODUCTION}

Although the study of conformal invariants of submanifolds received some attention in the classical geometry of surfaces, and later from Blaschke [B] and others, it remained almost unknown until 1965. In that year, Willmore [Wi1 proposed the study of a global conformal invariant for compact surfaces in Euclidean spaces, often called the Willmore functional, that associates to an immersion $\phi: \Sigma \rightarrow \mathbb{R}^{n}$ from a compact surface, the conformally invariant integral

$$
\mathcal{W}(\phi)=\int_{\Sigma}|H|^{2} d A
$$

where $H$ denotes the mean curvature vector of the immersion $\phi$ and $d A$ is the area measure of the induced metric. One easily obtains that the absolute minimum for $\mathcal{W}$ is $4 \pi$, which is attained only by the umbilical spheres. When the genus of $\Sigma$ is one (that is, when $\Sigma$ is a torus) the minimum value of $\mathcal{W}$ was conjectured by Willmore to be $2 \pi^{2}$ and to be reached only by (stereographic projection of) the Clifford torus. This conjecture remains open, although there are some good approaches which have been sources of new ideas, such as [Wi2], [LY], [K1], [K2], MRo. Very recently, A. Ros has solved the conjecture in case the torus is invariant under an antipodal symmetry $[\mathrm{R}$.

Besides the problem of the minimization of the Willmore functional $\mathcal{W}$, some authors have tried to determine its critical (not necessarily minimal) values and study

Received by the editors September 30, 1998 and, in revised form, March 15, 1999.

2000 Mathematics Subject Classification. Primary 53C40; Secondary 53A10, 53C28.

Key words and phrases. Willmore surface, minimal surface.

Research partially supported by a DGICYT grant PB97-0785.

(C)2000 American Mathematical Society 
the moduli spaces of $\mathcal{W}$-critical immersions in $\mathbb{R}^{n}$ (or in $\mathbb{S}^{n}$, which is the conformal compactification of Euclidean space). This kind of work was started by R. Bryant, who, in his papers Br1 and Br2, has shown that in the genus zero case methods of algebraic geometry are successful in order to classify Willmore immersions in $\mathbb{S}^{3}$, and that there exists a close relation between Willmore immersions and a class of minimal surfaces of Euclidean space $\mathbb{R}^{3}$ : those with finite total curvature and embedded planar ends. This has been explored by Kusner [K1] and has opened the way to a spinor consideration of the subject (see [KS]).

In this paper, using also a spinor approach, we study Willmore immersions of the two-sphere $\mathbb{S}^{2}=\mathbb{P}^{1}$ into the four-sphere $\mathbb{S}^{4}$ (in a more general context, Ejiri [E] obtained some partial results). In fact, by studying the conformal Gauss map of a conformal immersion $\phi: \mathbb{P}^{1} \rightarrow \mathbb{S}^{4}$ and its twistor lifting to the complex projective space $\mathbb{P}^{3}$, we shall prove that the situation is slightly different from the case where the ambient space was three-dimensional. In that case, Bryant proved that all the genus zero Willmore immersions came, via inverse stereographic projection, from minimal surfaces in $\mathbb{R}^{3}$. We shall see in Theorems 5 and 7 that

All the genus zero Willmore immersions in $\mathbb{S}^{4}$ are found in one of these two classes: those obtained, via inverse stereographic projection, from complete minimal surfaces of $\mathbb{R}^{4}$ with finite total curvature and embedded planar ends; and those produced, via the Penrose twistor fibration $\mathbb{P}^{3} \rightarrow \mathbb{S}^{4}$, from a rational curve in the complex projective space $\mathbb{P}^{3}$. Such a Willmore immersion belongs to both classes when it comes from a minimal surface which is complex for some complex structure on $\mathbb{R}^{4}$ or, equivalently, comes from a rational curve in some $\mathbb{P}^{2} \subset \mathbb{P}^{3}$.

As a consequence of this, we shall determine the critical values of the Willmore functional $\mathcal{W}$ for immersions of $\mathbb{P}^{1}$ in $\mathbb{S}^{4}$. We shall show in Theorem 6 that

If $\phi: \mathbb{P}^{1} \rightarrow \mathbb{S}^{4}$ is a Willmore immersion whose regular homotopy class is determined by the Whitney self-intersection number $q \in \mathbb{Z}$, then $\mathcal{W}(\phi)=$ $4 \pi(1+|q|+n)$ for some $n \in \mathbb{N}$. The minimum value $4 \pi(1+|q|)$ is attained if and only if $\phi$ is obtained, via Penrose fibration, from a rational curve in $\mathbb{P}^{3}$.

Finally, in Corollary 8, we shall study Willmore immersions with a small value of the Willmore functional, and see that the values $8 \pi$ and $12 \pi$ (forbidden when the ambient was $\mathbb{S}^{3}$ ) can be reached and characterize certain Whitney and Veronese immersions.

\section{Preliminaries}

Let $\Sigma$ be an oriented surface and denote by $J$ the complex structure on $\Sigma$ which is compatible with the given orientation. Consider a conformal immersion $\phi: \Sigma \rightarrow$ $\mathbb{S}^{4} \subset \mathbb{R}^{5}$ into the unit four-sphere, which will be endowed with its standard metric. The fact that $\phi$ is a conformal immersion can be expressed, in terms of a local isothermal parameter $z=x+i y$ on $\Sigma$, in the following way:

$$
\left|\phi_{z}\right|^{2}=\left\langle\phi_{z}, \phi_{\bar{z}}\right\rangle>0, \quad\left\langle\phi_{z}, \phi_{z}\right\rangle=0,
$$

where $\langle$,$\rangle means the \mathbb{C}$-linear extension of the usual Euclidean product of $\mathbb{R}^{5}$ to $\mathbb{C}^{5}=\mathbb{R}^{5} \otimes_{\mathbb{R}} \mathbb{C}$ and the partial derivatives with respect to $z$ and $\bar{z}$ are taken to be

$$
\partial=\frac{\partial}{\partial z}=\frac{\partial}{\partial x}-i \frac{\partial}{\partial y}, \quad \bar{\partial}=\frac{\partial}{\partial \bar{z}}=\frac{\partial}{\partial x}+i \frac{\partial}{\partial y} \text {. }
$$


We shall represent by $\sigma$ the second fundamental form of the immersion $\phi$ and by $H$ its mean curvature vector. So

$$
\phi_{z \bar{z}}=-\left|\phi_{z}\right|^{2} \phi+\left|\phi_{z}\right|^{2} H, \quad \phi_{z z}=\partial \log \left|\phi_{z}\right|^{2} \phi_{z}+\sigma_{z z},
$$

where $\sigma_{z z}=\sigma\left(\phi_{z}, \phi_{z}\right)$.

On the other hand, if $T \mathbb{S}^{4}$ is the tangent bundle of the sphere $\mathbb{S}^{4}$, the rank-four vector bundle $\phi^{*} T \mathbb{S}^{4}$ induced by the immersion $\phi$ on $\Sigma$ is a subbundle of the trivial bundle $\underline{\mathbb{R}^{5}}=\Sigma \times \mathbb{R}^{5}$. Then, we have the orthogonal decomposition

$$
\phi^{*} T \mathbb{S}^{4}=T \Sigma \oplus T^{\perp} \Sigma,
$$

where $T \Sigma$ and $T^{\perp} \Sigma$ are respectively the tangent bundle of $\Sigma$ and the normal bundle of the immersion $\phi$, and these two rank-two vector bundles on $\Sigma$ can be also viewed as subbundles of the trivial bundle $\mathbb{R}^{5}$. Then, the corresponding spaces of their smooth sections, denoted by $\Gamma(T \Sigma)$ and $\Gamma\left(T^{\perp} \Sigma\right)$, can be thought of as subspaces of $\Gamma\left(\underline{\mathbb{R}^{5}}\right)=C^{\infty}\left(\Sigma, \mathbb{R}^{5}\right)$.

Let $\bar{\nabla}$ be the connection on $\phi^{*} T \mathbb{S}^{4}$ induced by the Levi-Civita connection on $\mathbb{S}^{4}$. We have the decomposition

$$
\bar{\nabla}=\nabla+\nabla^{\perp}
$$

corresponding to (3), where $\nabla$ is the Levi-Civita connection of the metric induced on $\Sigma$ by $\phi$ and $\nabla^{\perp}$ is the normal connection. Since $\phi$ is conformal, we have that $\nabla J=0$, and so, via the Koszul-Malgrange theorem [KM], we obtain a unique holomorphic structure on the complex line bundle $T \Sigma$. It is clear that this structure is nothing but that of $\kappa^{*}$, the dual of the canonical line bundle $\kappa$ on the Riemann surface $\Sigma$.

We may also define another almost complex structure on the normal bundle $T^{\perp} \Sigma$, which will also be denoted by $J$, in the following way:

$$
J e_{3}=-e_{4},
$$

where $\left\{e_{1}, e_{2}=J e_{1}, e_{3}, e_{4}\right\}$ is an oriented orthonormal local frame on $\phi^{*} T \mathbb{S}^{4}$ and $\left\{e_{1}, e_{2}\right\}$ is a local frame on $T \Sigma$. It is easy to check that also $\nabla^{\perp} J=0$, and so the aforementioned Koszul-Malgrange theorem gives a unique structure of a holomorphic line bundle on $T^{\perp} \Sigma$, such that a section $\xi \in \Gamma\left(T^{\perp} \Sigma\right)$ is holomorphic if and only if

$$
\nabla_{J v}^{\perp} \xi=J \nabla_{v}^{\perp} \xi \quad v \in T \Sigma .
$$

We shall represent by $N$ this holomorphic line bundle on the Riemann surface $\Sigma$. In the case where $\Sigma$ is compact, the Chern number $c(N)$ of this line bundle $N$ is the Euler characteristic of the normal bundle $T^{\perp} \Sigma$ of the immersion $\phi$, and so

$$
c(N)=\frac{1}{2 \pi} \int_{\Sigma} K^{\perp} d A
$$

where $K^{\perp}$ is the normal curvature of the immersion given by

$$
K^{\perp}=R^{\perp}\left(e_{1}, e_{2}, e_{3}, e_{4}\right),
$$

$R^{\perp}$ is the curvature tensor of $\nabla^{\perp}$ and $d A$ is the area element of the induced metric.

Let $T_{\mathbb{C}} \mathbb{S}^{4}=T \mathbb{S}^{4} \otimes_{\mathbb{R}} \mathbb{C}$ be the complexified tangent bundle of the four-sphere. The induced complex bundle $\phi^{*} T_{\mathbb{C}} \mathbb{S}^{4}$ can be considered as a subbundle of the complex trivial bundle $\underline{\mathbb{C}^{5}}=\underline{\mathbb{R}^{5}} \otimes_{\mathbb{R}} \mathbb{C}$ on $\Sigma$. Then, we have the complex version of (3)

$$
\phi^{*} T_{\mathbb{C}} \mathbb{S}^{4}=T_{\mathbb{C}} \Sigma \oplus T_{\mathbb{C}}^{\perp} \Sigma .
$$


We shall continue to denote by $\bar{\nabla}, \nabla, \nabla^{\perp}, J$ and $\langle$,$\rangle the corresponding \mathbb{C}$-linear extensions of these geometric objects. Thus, the almost complex structures $J$ in $T_{\mathbb{C}} \Sigma$ and $T_{\mathbb{C}}^{\perp} \Sigma$ yield splittings of the corresponding complex bundles into $(1,0)$ and $(0,1)$ subbundles which are eigenbundles for the eigenvalues $+i$ and $-i$. In fact, we have

$$
T_{\mathbb{C}} \Sigma=T_{\mathbb{C}} \Sigma^{(1,0)} \oplus T_{\mathbb{C}} \Sigma^{(0,1)}, \quad T_{\mathbb{C}}^{\perp} \Sigma=T_{\mathbb{C}}^{\perp} \Sigma^{(1,0)} \oplus T_{\mathbb{C}}^{\perp} \Sigma^{(0,1)}
$$

These splittings are preserved by the corresponding connections $\nabla$ and $\nabla^{\perp}$, and give subbundles where the $\mathbb{C}$-bilinear product $\langle$,$\rangle is null. It is clear that the$ holomorphic line bundles $T \Sigma$ and $N$ which we have defined by using the KoszulMalgrange theorem are respectively isomorphic to $T_{\mathbb{C}} \Sigma^{(1,0)}$ and to $T_{\mathbb{C}}^{\perp} \Sigma^{(1,0)}$, in such a way that if we represent by $\partial$ and $\bar{\partial}$ the operators

$$
\partial \xi=\left(\nabla_{\frac{\partial}{\partial z}}^{\perp} \xi\right) \otimes d z, \quad \bar{\partial} \xi=\left(\nabla_{\frac{\partial}{\partial \bar{z}}}^{\perp} \xi\right) \otimes d \bar{z}
$$

where $\xi \in \Gamma\left(T_{\mathbb{C}}^{\perp} \Sigma\right)$, then $\xi$ is holomorphic if and only if $\bar{\partial} \xi=0$. We shall also denote by $\partial$ and $\bar{\partial}$ the corresponding operators on any complex vector bundle on $\Sigma$ of the form $T \Sigma \otimes V$ or $T^{\perp} \Sigma \otimes V$, where $V$ is a given complex vector space. Now consider the Euclidean space $\mathbb{R}^{5}$ as the hyperplane $x_{1}=0$ in $\mathbb{R}^{6}$ and let $A=(1,0, \ldots, 0) \in \mathbb{R}^{6}$. So

$$
\mathbb{R}^{6}=\langle A\rangle \oplus \mathbb{R}^{5}
$$

where $\langle A\rangle$ is the line spanned by the vector $A$. We shall endow this real vector space $\mathbb{R}^{6}$ with the Lorentzian metric (that we shall also denote by $\langle$,$\rangle ) A$ becoming a unit timelike vector orthogonal to $\mathbb{R}^{5}$ and inducing on this $\mathbb{R}^{5}$ the usual Euclidean metric. We shall use the notation $\mathbb{R}_{1}^{6}$ in order to point out the presence of this indefinite metric. Complexifying (4), we can also view the complex space $\mathbb{C}^{5}$ as the hyperplane $z_{1}=0$ in

$$
\mathbb{C}^{6}=\langle A\rangle \oplus \mathbb{C}^{5}
$$

where now $\langle A\rangle$ stands for the complex line spanned by $A$. Represent again by $\langle$,$\rangle the corresponding \mathbb{C}$-bilinear extension of the Lorentzian product above, and denote by $\mathbb{C}_{1}^{6}$ the corresponding complex metric space. Notice that, if $u, v \in \mathbb{C}_{1}^{6}$, the product $\langle u, \bar{v}\rangle$ yields an indefinite Hermitian product on $\mathbb{C}_{1}^{6}$.

Associated to our conformal immersion $\phi: \Sigma \rightarrow \mathbb{S}^{4}$, we may define a map $\mathcal{R}: T_{\mathbb{C}}^{\perp} \Sigma \rightarrow \mathbb{C}_{1}^{6}$ by

$$
\mathcal{R}(\xi)=\langle H, \xi\rangle(A+\phi)+\xi, \quad \xi \in T_{\mathbb{C}}^{\perp} \Sigma .
$$

This map is linear in $\xi$, and so it provides us a section of $\left(T_{\mathbb{C}}^{\perp} \Sigma\right)^{*} \otimes \mathbb{C}_{1}^{6}$. Let $R$ the $(1,0)$-part of $\mathcal{R}$ (that is the part vanishing on $\left.T_{\mathbb{C}}^{\perp} \Sigma^{(0,1)}\right)$. Because

$$
\mathcal{R}(\bar{\xi})=\overline{\mathcal{R}(\xi)} \text { for all } \xi \in T_{\mathbb{C}}^{\perp} \Sigma,
$$

the corresponding $(0,1)$-part of $\mathcal{R}$ is $\bar{R}$. Then

$$
R \in \Gamma\left(N^{*} \otimes \mathbb{C}_{1}^{6}\right), \quad \bar{R} \in \Gamma\left(\bar{N}^{*} \otimes \mathbb{C}_{1}^{6}\right),
$$

where $N^{*}$ means the dual line bundle of $N$. By using the $\mathbb{C}$-bilinear product of $\mathbb{C}_{1}^{6}$, we obtain sections

$$
\langle R, R\rangle \in \Gamma\left(N^{*} \otimes N^{*}\right), \quad\langle\bar{R}, \bar{R}\rangle \in \Gamma\left(\bar{N}^{*} \otimes \bar{N}^{*}\right), \quad\langle R, \bar{R}\rangle \in \Gamma\left(N^{*} \otimes \bar{N}^{*}\right) .
$$


We must remark that the line bundle $N^{*} \otimes \bar{N}^{*}$ on $\Sigma$ is holomorphically trivial provided that the Hermitian product

$$
\left\langle,{ }^{-}\right\rangle: N^{*} \otimes \bar{N}^{*} \rightarrow \mathbb{C}
$$

is a parallel section of it. Hence, from now on, we shall identify the line bundles $\bar{N}$ and $N^{*}$ when convenient. From the definition (5), one immediately has

$$
\langle R, R\rangle=0, \quad\langle\bar{R}, \bar{R}\rangle=0, \quad\langle R, \bar{R}\rangle=1 .
$$

These equalities imply that the section $R$ of the vector bundle $N^{*} \otimes \mathbb{C}_{1}^{6}$ determines a map

$$
\mathcal{G}: \Sigma \longrightarrow \mathbb{Q}_{1}^{4} \subset \mathbb{P}_{1}^{5}, \quad \mathcal{G}=[R],
$$

where $[R]$ means the complex line spanned by $R(\xi)$ in $\mathbb{C}_{1}^{6}$, with $\xi$ being any local section of $N$, and

$$
\mathbb{P}_{1}^{5}=\left\{[z] \mid z \in \mathbb{C}_{1}^{6},\langle z, \bar{z}\rangle>0\right\}
$$

is the indefinite complex projective space (see [Wo, p. 378), defined as the space of spacelike complex lines in the metric complex vector space $\mathbb{C}_{1}^{6}$. The first equality in (6) says that the image of this map $\mathcal{G}$ lies in the complex hypersurface

$$
\mathbb{Q}_{1}^{4}=\left\{[z] \mid z \in \mathbb{C}_{1}^{6},\langle z, \bar{z}\rangle>0,\langle z, z\rangle=0\right\},
$$

which is the indefinite analogue of the complex hyperquadric in the usual complex projective space. As in the definite case, the complex manifold $\mathbb{P}_{1}^{5}$, which is biholomorphic to an open set of $\mathbb{P}^{5}$, can be endowed with an indefinite (index two) Fubini-Study metric

$$
d s_{F S}^{2}=\frac{|d z \wedge z|^{2}}{|z|^{4}}=\frac{|z|^{2}|d z|^{2}-|\langle z, d \bar{z}\rangle|^{2}}{|z|^{4}}
$$

and becomes an indefinite Kähler manifold with constant holomorphic sectional curvature 4 . In this way, the hyperquadric $\mathbb{Q}_{1}^{4}$ is an Einstein hypersurface and has all the analogous properties that the usual hyperquadric has in the definite case (see [MR]). It is an indefinite Kähler manifold with real dimension eight and real index two, which is isometric to the non-compact symmetric space

$$
\frac{S O(5,1)}{S O(3,1) \times S O(2)}
$$

consisting of the oriented spacelike 2-planes in the Lorentzian space $\mathbb{R}_{1}^{6}$. The map $\mathcal{G}: \Sigma \rightarrow \mathbb{Q}_{1}^{4}$ is a generalization of the so-called conformal Gauss map, defined by Blaschke [B1] and rediscovered by Bryant [Br1] in the case of conformal immersions into the three-sphere, and it has already considered when the ambient space is four-dimensional by Ejiri in E].

If we compute directly, using (2), we have that, for each $\xi \in \Gamma(N)$,

$$
\left(\nabla_{\frac{\partial}{\partial z}} R\right)(\xi)=R(\xi)_{z}-R\left(\nabla_{\frac{\partial}{\partial z}}^{\perp} \xi\right)=\left\langle\nabla_{\frac{\partial}{\partial z}}^{\perp} H, \xi\right\rangle(A+\phi)-\frac{\left\langle\sigma_{z z}, \xi\right\rangle}{\left|\phi_{z}\right|^{2}} \phi_{\bar{z}}
$$

where $\nabla$ is the natural connection on $\left(T^{\perp} \Sigma\right)^{*}$. Then, from this (8) and (5), since

$$
\partial R=\left(\nabla_{\frac{\partial}{\partial z}} R\right) \otimes d z
$$

we get

$$
\langle\partial R, R\rangle=0, \quad\langle\partial \bar{R}, \bar{R}\rangle=0, \quad\langle\partial R, \bar{R}\rangle=0, \quad\langle\partial \bar{R}, R\rangle=0 .
$$


Moreover, as the immersion $\phi$ is conformal, taking (1) into account, we have

$$
\begin{gathered}
\langle\partial R, \partial R\rangle=0, \quad\langle\partial \bar{R}, \partial \bar{R}\rangle=0, \quad\langle\partial R, \partial \bar{R}\rangle=0, \\
\langle\partial R, \bar{\partial} R\rangle(\xi, \eta)=\frac{\left\langle\sigma_{z z}, \xi\right\rangle\left\langle\sigma_{\overline{z z}}, \eta\right\rangle}{\left|\phi_{z}\right|^{2}} \\
|\partial R|^{2}(\xi, \bar{\xi})=\frac{\left|\left\langle\sigma_{z z}, \xi\right\rangle\right|^{2}}{\left|\phi_{z}\right|^{2}}|d z|^{2}, \quad|\bar{\partial} R|^{2}(\xi, \bar{\xi})=\frac{\left|\left\langle\sigma_{z z}, \bar{\xi}\right\rangle\right|^{2}}{\left|\phi_{z}\right|^{2}}|d z|^{2},
\end{gathered}
$$

where $\xi, \eta \in N$.

The third of these equalities implies, together with the definition of the FubiniStudy metric on $\mathbb{P}_{1}^{5}$, that the conformal Gauss map $\mathcal{G}$ is conformal. From (9) and the last two equalities in (10), one can see that the metric on $\Sigma$ induced by $\mathcal{G}$ is given by

$$
4 \mathcal{G}^{*} d s_{F S}^{2}=\frac{|R \wedge \partial R|^{2}}{|R|^{4}}+\frac{|R \wedge \bar{\partial} R|^{2}}{|R|^{4}}=|\partial R|^{2}+|\bar{\partial} R|^{2} .
$$

Then, we obtain

$$
\mathcal{G}^{*} d s_{F S}^{2}=\frac{1}{4} \frac{\left|\sigma_{z z}\right|^{2}}{\left|\phi_{z}\right|^{2}}|d z|^{2}=\frac{1}{4}\left(1+|H|^{2}-K\right)\left|\phi_{z}\right|^{2}|d z|^{2},
$$

where $K$ is the Gauss curvature of the metric $\frac{1}{2}\left|\phi_{z}\right|^{2}|d z|^{2}$ induced on $\Sigma$ from the immersion $\phi$. Hence, the conformal Gauss map is an immersion away from the points with $\sigma_{z z}=0$; that is, it has singularities at the umbilical points of $\phi$. Moreover, $\mathcal{G}$ is holomorphic (respectively antiholomorphic) at the points satisfying $\left\langle\sigma_{z z}, \bar{\xi}\right\rangle=0$, for any $\bar{\xi} \in N$ (respectively $\left\langle\sigma_{z z}, \xi\right\rangle=0$ ); that is, $\sigma_{z z} \in \bar{N}=T_{\mathbb{C}}^{\perp} \Sigma^{(0,1)}$ (respectively $\sigma_{z z} \in N=T_{\mathbb{C}}^{\perp} \Sigma^{(1,0)}$ ).

In the case where $\Sigma$ is compact, integrating the equality above, one has the area of the image of this conformal Gauss map:

$$
2 A(\mathcal{G})=\int_{\Sigma}\left(1+|H|^{2}-K\right) d A=\mathcal{W}(\phi)-2 \pi \chi(\Sigma),
$$

where $\chi(\Sigma)$ is the Euler characteristic of the surface $\Sigma$.

On the other hand, also from (10), we conclude that

$$
|\partial R|^{2}-|\bar{\partial} R|^{2}=\frac{1}{\left|\phi_{z}\right|^{2}}\left(\left|\left\langle\sigma_{z z}, \xi\right\rangle\right|^{2}-\left|\left\langle\sigma_{z z}, \bar{\xi}\right\rangle\right|^{2}\right)|d z|^{2},
$$

where $\xi$ is any local section of $N$ with $|\xi|^{2}=\langle\xi, \bar{\xi}\rangle=1$. Using the Ricci equation for the immersion $\phi$, we deduce that

$$
|\partial R|^{2}-|\bar{\partial} R|^{2}=R^{\perp}\left(\phi_{\bar{z}}, \phi_{z}, \xi, \bar{\xi}\right)|d z|^{2}=K^{\perp}\left|\phi_{z}\right|^{2}|d z|^{2} .
$$

When $\Sigma$ is compact, if we integrate this last equality, we compute the degree of the conformal Gauss map $\mathcal{G}$, as a map from $\Sigma$ to the indefinite projective space $\mathbb{P}_{1}^{5}$ (which has the same homotopy type that $\mathbb{P}^{4}$, and so $H_{2}\left(\mathbb{P}_{1}^{5}, \mathbb{Z}\right)=\mathbb{Z}$ ). In fact,

$$
4 \pi \operatorname{deg} \mathcal{G}=\int_{\Sigma}\left(|\partial R|^{2}-|\bar{\partial} R|^{2}\right) d x d y=4 \pi c(N)
$$

Remark 1. The expressions above, computing the area and the degree of the conformal Gauss map $\mathcal{G}$ of the immersion $\phi$, directly imply that

$$
\mathcal{W}(\phi)-2 \pi \chi(\Sigma) \geq 2 \pi|c(N)|,
$$

the equality holding if and only if the conformal Gauss map is either a holomorphic or a antiholomorphic map from $\Sigma$ into the indefinite Kähler manifold $\mathbb{Q}_{1}^{4} \subset \mathbb{P}_{1}^{5}$. 
This inequality was first found by Wintgen Win and Weiner We, and the case of equality was studied by Friedrich $[\mathbf{F}$ in terms of twistor liftings. But, in the following section, we shall show that the conformal Gauss map $\mathcal{G}$ of the immersion $\phi$ is holomorphic if and only if its twistor lifting is holomorphic.

\section{TWISTOR LIFTINGS}

It is well known (see, for example, $[\underline{S}]$ ) that the twistor space (that is, the space of the almost Hermitian structures which are compatible with a given orientation) of the four-sphere $\mathbb{S}^{4}$ (with either of the two possible orientations) can be identified with the complex projective space $\mathbb{P}^{3}$ with its complex standard structure. We may associate to our conformal immersion $\phi: \Sigma \rightarrow \mathbb{S}^{4}$ the so-called twistor lifting of $\phi$, $\mathcal{T}: \Sigma \rightarrow \mathbb{P}^{3}$, which maps each point $p \in \Sigma$ to the almost complex structure on the tangent space $T_{\phi(p)} \mathbb{S}^{4}$ determined by the two almost complex structures $J$ which we have already found on $T_{p} \Sigma$ and $T_{p}^{\perp} \Sigma$ (see [ES] or [F]).

One of the natural ways to describe the twistor lifting of a conformal map from a Riemann surface to the four-sphere is based on the fact that the complex projective three-space $\mathbb{P}^{3}$ can be identified with the space of null two-planes of the complex vector space $\mathbb{C}^{5}$, which is a symmetric space isometric to

$$
\frac{S O(5)}{U(2)} \cong \frac{U(4)}{U(3) \times U(1)} \cong \mathbb{P}^{3} .
$$

Moreover, if $P \subset \mathbb{C}^{5}$ is such a two-plane and $\{u, v\}$ is a basis of it, the map $P \mapsto[u \wedge v] \in \mathbb{P}\left(\wedge^{2} \mathbb{C}^{5}\right)$ is an embedding

$$
\mathbb{P}^{3} \longrightarrow \mathbb{P}^{9} \cong \mathbb{P}\left(\bigwedge^{2} \mathbb{C}^{5}\right)
$$

It is not difficult to check that this is the Veronese embedding, which is realized through all the homogeneous second degree polynomials in the homogeneous coordinates of $\mathbb{P}^{3}$. In fact, given our conformal immersion $\phi: \Sigma \rightarrow \mathbb{S}^{4}$, we consider the section

$$
T=\left(\phi_{\bar{z}} \wedge \xi\right) \otimes d \bar{z} \in \Gamma\left(\bar{\kappa} \otimes N^{*} \otimes \bigwedge^{2} \mathbb{C}^{5}\right),
$$

which induces a map from the Riemann surface $\Sigma$ into the complex projective space $\mathbb{P}\left(\bigwedge^{2} \mathbb{C}^{5}\right) \cong \mathbb{P}^{9}$. This map $\mathcal{T}=[T]$ is the twistor lifting of $\phi$. (Notice that, as the antipodal map of $\mathbb{S}^{4}$ reverses the orientation, the twistor lifting of the antipodal of the immersion $\phi$ is given by means of the section $\left(\phi_{\bar{z}} \wedge \bar{\xi}\right) \otimes d \bar{z}$.) It is clear from (2) that

$$
\partial T=\left(-\left|\phi_{z}\right|^{2} \phi \wedge \xi+\left|\phi_{z}\right|^{2}\langle H, \xi\rangle \bar{\xi} \wedge \xi+\langle H, \xi\rangle \phi_{\bar{z}} \wedge \phi_{z}\right) \otimes d z \otimes d \bar{z}
$$

From that, one obtains

$$
\frac{|\partial T \wedge T|^{2}}{|T|^{4}}=\frac{|\partial T|^{2}}{|T|^{2}}=\left|\phi_{z}\right|^{2}\left(1+|H|^{2}\right)|d z|^{2} .
$$

Analogously, we can check that

$$
\bar{\partial} T=\left(\left\langle\sigma_{\overline{z z}}, \xi\right\rangle \bar{\xi} \wedge \xi+\frac{\left\langle\sigma_{\overline{z z}}, \xi\right\rangle}{\left|\phi_{z}\right|^{2}} \phi_{\bar{z}} \wedge \phi_{z}\right) \otimes d \bar{z} \otimes d \bar{z}
$$

Thus, one gets

$$
\frac{|\bar{\partial} T \wedge T|^{2}}{|T|^{4}}=\frac{|\bar{\partial} T|^{2}}{|T|^{2}}=2 \frac{\left|\left\langle\sigma_{z z}, \bar{\xi}\right\rangle\right|^{2}}{\left|\phi_{z}\right|^{2}}=\left|\phi_{z}\right|^{2}\left(1+|H|^{2}-K-K^{\perp}\right)|d z|^{2},
$$


where $\xi$ was a unit local section of $N$. A first consequence of these equalities and (10) is

Lemma 1. The twistor lifting $\mathcal{T}: \Sigma \rightarrow \mathbb{P}^{3}$ of a conformal immersion $\phi: \Sigma \rightarrow \mathbb{S}^{4}$ is holomorphic at exactly the same points where its conformal Gauss map $\mathcal{G}: \Sigma \rightarrow \mathbb{Q}_{1}^{4}$ is holomorphic. (The twistor lifting of the antipodal immersion $-\phi$ is holomorphic at exactly those points where the conformal Gauss map is antiholomorphic.)

We may obtain a second consequence of those computations. Now, we easily check that

$$
\frac{|\partial T \wedge T|^{2}}{|T|^{4}}-\frac{|\bar{\partial} T \wedge T|^{2}}{|T|^{4}}=\left|\phi_{z}\right|^{2}\left(K+K^{\perp}\right) .
$$

So, if $\Sigma$ is compact, we integrate this equality and get

$$
\operatorname{deg}\left(\mathcal{T}: \Sigma \rightarrow \mathbb{P}^{9}\right)=\chi(\Sigma)+c(N)
$$

But the image of $\mathcal{T}$ lies in $\mathbb{P}^{3}$ and the embedding $\mathbb{P}^{3} \subset \mathbb{P}^{9}$ induces a degree two map on the homology. So, we finally have

$$
\chi(\Sigma)+c(N)=2 \operatorname{deg} \mathcal{T} .
$$

Then, since $\chi(\Sigma)$ is an even number, the Euler number of the normal bundle of the immersion $\phi$ is also an even number,

$$
c(N)=2(\operatorname{deg} \mathcal{T}+g-1),
$$

where $g$ is the genus of the compact surface $\Sigma$.

Remark 2. Notice that Lashof and Smale [LS showed that the Euler number of the normal bundle of an immersion $\phi: \Sigma \rightarrow \mathbb{S}^{4}$ is $c(N)=2 q$, where $q \in \mathbb{Z}$ is the self-intersection number of the immersion. Then $q=\operatorname{deg} \mathcal{T}+g-1$. Moreover, in Hi], Theorem 8.3, Hirsch proved that the number $c(N)=2 q$ classifies the regular homotopy classes of immersion from a two-sphere into the four-sphere.

\section{WILLMORE SURFACES IN THE FOUR-SPHERE}

We proceed now to compute the tension field of the conformal Gauss map $\mathcal{G}$ of the conformal immersion $\phi$. For that, we must look for

$$
\bar{\partial} \partial R=\nabla_{\frac{\partial}{\partial z}} \nabla_{\frac{\partial}{\partial z}} R \otimes d z \otimes d \bar{z} \in \Gamma\left(\kappa^{*} \otimes \bar{\kappa}^{*} \otimes N\right) \cong \Gamma(N) .
$$

Let $\xi \in N$. Then, by using (2), (8) and the Codazzi equation for the immersion $\phi$, we have

$$
\left(\nabla_{\frac{\partial}{\partial \bar{z}}} \nabla_{\frac{\partial}{\partial z}} R\right)(\xi)=\left\langle\nabla_{\frac{\partial}{\partial \bar{z}}}^{\frac{\partial}{\partial}} \nabla_{\frac{\partial}{\partial z}}^{\perp} H, \xi\right\rangle(A+\phi)-\frac{\left\langle\sigma_{z z}, \xi\right\rangle}{\left|\phi_{z}\right|^{2}} \sigma_{\overline{z z}} .
$$

But, if we recall the definition (5) of the section $\mathcal{R} \in \Gamma\left(T_{\mathbb{C}}^{\perp} \Sigma \otimes \mathbb{C}_{1}^{6}\right)$, we deduce that

$$
\left(\nabla_{\frac{\partial}{\partial \bar{z}}} \nabla_{\frac{\partial}{\partial z}} R\right)(\xi)=\left\langle\nabla_{\frac{\partial}{\partial z}}^{\perp} \nabla_{\frac{\partial}{\partial z}}^{\perp} H+\frac{\left\langle H, \sigma_{\overline{z z}}\right\rangle}{\left|\phi_{z}\right|^{2}} \sigma_{z z}, \xi\right\rangle(A+\phi)-\frac{\left\langle\sigma_{z z}, \xi\right\rangle}{\left|\phi_{z}\right|^{2}} \mathcal{R}\left(\sigma_{\overline{z z}}\right) .
$$

As a conclusion, we obtain

$$
\bar{\partial} \partial R=s \otimes R+t \otimes \bar{R} \Longleftrightarrow \nabla_{\frac{\partial}{\partial z}}^{\perp} \nabla_{\frac{\partial}{\partial z}}^{\perp} H+\frac{1}{\left|\phi_{z}\right|^{2}}\left\langle H, \sigma_{\overline{z z}}\right\rangle \sigma_{z z}=0,
$$

because the field on the left side is real and where $s$ and $t$ are necessarily sections in the line bundles $\kappa \otimes \bar{\kappa} \otimes N^{*} \otimes \bar{N}^{*}$ and $\kappa \otimes \bar{\kappa} \otimes N^{*} \otimes N^{*}$, respectively (recall that 
$N^{*} \otimes \bar{N}^{*}$ is holomorphically trivial). But the right side in (13) is just the Euler equation for the Willmore functional (see for example $\mathrm{E}$ ), and in the left side, from (9), we have that the sections $s$ and $t$ must be

$$
s=-|\partial R|^{2}=-\langle\partial R, \overline{\partial R}\rangle, \quad t=-\langle\partial R, \bar{\partial} R\rangle .
$$

From these considerations and the definition (7) the following result arises.

Proposition 2. Let $\phi: \Sigma \rightarrow \mathbb{S}^{4}$ be a conformal immersion from a Riemann surface into the four-sphere, $R$ the corresponding section of the complex vector bundle $N^{*} \otimes$ $\mathbb{C}_{1}^{6}$ defined in (5), and $\mathcal{G}=[R]: \Sigma \rightarrow \mathbb{Q}_{1}^{4}$ its conformal Gauss map. The following assertions are equivalent:

1. $\phi$ is a Willmore immersion, that is, it is a critical point for the Willmore functional.

2. $\mathcal{G}$ is harmonic and, so, a minimal immersion away from its singular points.

3. $\bar{\partial} \partial R=-|\partial R|^{2} \otimes R-\langle\partial R, \bar{\partial} R\rangle \otimes \bar{R}$ (or, equivalently, $\bar{\partial} \partial \bar{R}=-|\partial \bar{R}|^{2} \otimes \bar{R}-$ $\langle\partial \bar{R}, \overline{\partial R}\rangle \otimes R)$.

Remark 3. The equivalence between the first two assertions in Proposition 2 above was already pointed out in $[\mathrm{E}]$ in a more general context, and it is a generalization of the corresponding property proved by Bryant [Br1] in the case of immersions into the three-sphere.

Remark 4. Notice that, if $\phi$ is a Willmore immersion, as $\mathcal{G}$ must be conformal and harmonic, it is also an analytic map. Hence, from (11), either $\sigma_{z z}$ is identically zero and (so $\phi$ is an umbilical embedding from a two-sphere into $\mathbb{S}^{4}$ as a two-dimensional equator), or $\sigma_{z z}$ vanishes only on a (closed) set with empty interior. Then, in this non-umbilical case, the set $\Sigma^{\prime}$ consisting of the non-umbilical points of the Willmore immersion $\phi$ is an open dense set.

From now on, we shall suppose that the immersion $\phi: \Sigma \rightarrow \mathbb{S}^{4}$ is a Willmore immersion. So, we shall be able to utilize each statement in Proposition 2 above. We shall start by defining three sections of some holomorphic line bundles on the Riemann surface $\Sigma$, which, after considering suitable trivializations, will be respectively a 3-differential, a 4-differential and a 8-differential on the Riemann surface $\Sigma$. In fact, we put

$$
\begin{gathered}
\alpha=\left\langle\partial^{2} R, \partial \bar{R}\right\rangle \in \Gamma\left(\kappa^{3} \otimes N^{*} \otimes \bar{N}^{*}\right), \\
\beta=\left\langle\partial^{2} R, \partial^{2} \bar{R}\right\rangle \in \Gamma\left(\kappa^{4} \otimes N^{*} \otimes \bar{N}^{*}\right), \\
\gamma=\left\langle\partial^{2} R, \partial^{2} R\right\rangle \otimes\left\langle\partial^{2} \bar{R}, \partial^{2} \bar{R}\right\rangle \in \Gamma\left(\kappa^{8} \otimes N^{*} \otimes \bar{N}^{*} \otimes N^{*} \otimes \bar{N}^{*}\right) .
\end{gathered}
$$

Now, we want to study the effect that the $\bar{\partial}$-operator produces on these three differentials. With respect to $\alpha$, we have

$$
\bar{\partial} \alpha=\left\langle\bar{\partial} \partial^{2} R, \partial \bar{R}\right\rangle+\left\langle\partial^{2} R, \bar{\partial} \partial \bar{R}\right\rangle .
$$

But, since we are assuming $\phi$ to be a Willmore immersion, from 3) in Proposition 2, we obtain that $\left\langle\partial^{2} R, \bar{\partial} \partial R\right\rangle=0$, because, taking $\partial$-derivatives in (9) and using (10), one gets

$$
\left\langle\partial^{2} R, R\right\rangle=0, \quad\left\langle\partial^{2} R, \bar{R}\right\rangle=0, \quad\left\langle\partial^{2} \bar{R}, \bar{R}\right\rangle=0 .
$$

So, we deduce finally that

$$
\bar{\partial} \alpha=\left\langle\bar{\partial} \partial^{2} R, \partial \bar{R}\right\rangle .
$$


Now, the Ricci equation for the line bundle $\kappa \otimes N^{*}$ leads us to

$$
\bar{\partial} \partial^{2} R=\partial \bar{\partial} \partial R-\left(K^{\perp}+K\right)\left|\phi_{z}\right|^{2} \partial R \otimes d z \otimes d \bar{z} .
$$

So, from (10), we arrive at

$$
\bar{\partial} \alpha=\langle\partial \bar{\partial} \partial R, \partial \bar{R}\rangle .
$$

But, from Proposition 2, $\bar{\partial} \partial R \in \operatorname{span}\{R, \bar{R}\}$, and so $\partial \bar{\partial} \partial R \in \operatorname{span}\{R, \bar{R}, \partial R, \partial \bar{R}\}$. Hence, using again (9) and (10), we have $\bar{\partial} \alpha=0$, so

$$
\alpha \in H^{0}\left(\Sigma, \kappa^{3} \otimes N^{*} \otimes \bar{N}^{*}\right) \cong H^{0}\left(\Sigma, \kappa^{3}\right) ;
$$

that is, $\alpha$ is a holomorphic three-differential on the Riemann surface $\Sigma$, provided that $\phi$ is a Willmore immersion.

With respect to the 4 -differential $\beta$, we obtain that

$$
\bar{\partial} \beta=\left\langle\bar{\partial} \partial^{2} R, \partial^{2} \bar{R}\right\rangle+\left\langle\partial^{2} R, \bar{\partial} \partial^{2} \bar{R}\right\rangle .
$$

But the Ricci equation (16) for $\kappa \otimes N^{*}$ and the corresponding one for $\kappa \otimes \bar{N}^{*}$, together with the fact that from (10) we have

$$
0=\partial\langle\partial R, \partial \bar{R}\rangle=\left\langle\partial^{2} R, \partial \bar{R}\right\rangle+\left\langle\partial R, \partial^{2} \bar{R}\right\rangle,
$$

give us

$$
\bar{\partial} \beta=\left\langle\partial \bar{\partial} \partial R, \partial^{2} \bar{R}\right\rangle+\left\langle\partial \bar{\partial} \partial \bar{R}, \partial^{2} R\right\rangle-2\left|\phi_{z}\right|^{2} K^{\perp} \alpha \otimes d z \otimes d \bar{z}
$$

Now, as a consequence of 3) in Proposition 2, one has

$$
\partial \bar{\partial} \partial R+|\partial R|^{2} \otimes \partial R \in \operatorname{span}\{R, \bar{R}, \partial \bar{R}\}
$$

and an analogue for $\bar{R}$. Thus, we finally get

$$
\bar{\partial} \beta=\left|\phi_{z}\right|^{2} K^{\perp} \alpha \otimes d z \otimes d \bar{z} .
$$

Finally, taking the $\bar{\partial}$-derivative for the 8 -differential $\gamma$, one finds that

$$
\bar{\partial} \gamma=2\left\langle\bar{\partial} \partial^{2} R, \partial^{2} R\right\rangle \otimes\left\langle\partial^{2} \bar{R}, \partial^{2} \bar{R}\right\rangle+2\left\langle\partial^{2} R, \partial^{2} R\right\rangle \otimes\left\langle\bar{\partial} \partial^{2} \bar{R}, \partial^{2} \bar{R}\right\rangle .
$$

But (16) and the analogue for $\bar{R}$, together with (10), imply

$$
\begin{aligned}
& \left\langle\bar{\partial} \partial^{2} R, \partial^{2} R\right\rangle=\left\langle\partial \bar{\partial} \partial R, \partial^{2} R\right\rangle, \\
& \left\langle\bar{\partial} \partial^{2} \bar{R}, \partial^{2} \bar{R}\right\rangle=\left\langle\partial \bar{\partial} \partial \bar{R}, \partial^{2} \bar{R}\right\rangle .
\end{aligned}
$$

Using again 3 in Proposition 2, we conclude that

$$
\partial \bar{\partial} \partial R+\langle\partial R, \bar{\partial} R\rangle \otimes \partial R \in \operatorname{span}\{R, \bar{R}, \partial R\}
$$

and correspondingly for $\bar{R}$. Then, we obtain

$$
\bar{\partial} \gamma=2\left(\langle\partial \bar{R}, \overline{\partial R}\rangle \otimes\left\langle\partial^{2} R, \partial^{2} R\right\rangle-\langle\partial R, \bar{\partial} R\rangle \otimes\left\langle\partial^{2} \bar{R}, \partial^{2} \bar{R}\right\rangle\right) \otimes \alpha .
$$

Now, we have already calculated in (17), (18) and (19) the $\bar{\partial}$-derivatives of the three differentials $\alpha, \beta$ and $\gamma$ on the Riemann surface $\Sigma$. The following result is immediate from that.

Proposition 3. Let $\phi: \mathbb{P}^{1} \rightarrow \mathbb{S}^{4}$ be a conformal Willmore immersion of a genus zero surface into the four-sphere, and denote by $R$ the section of the complex vector bundle $N^{*} \otimes \mathbb{C}_{1}^{6}$ defined in (5). Then

$$
\left\langle\partial^{2} R, \partial \bar{R}\right\rangle=0, \quad\left\langle\partial^{2} R, \partial^{2} \bar{R}\right\rangle=0, \quad\left\langle\partial^{2} R, \partial^{2} R\right\rangle \otimes\left\langle\partial^{2} \bar{R}, \partial^{2} \bar{R}\right\rangle=0 .
$$

Proof. The point is that on $\mathbb{P}^{1}$ not only is $\alpha$ holomorphic, but also it vanishes; thus $\beta$ and $\gamma$ are holomorphic, and so vanish as well. 
Remark 5. The 3-differential $\alpha$ on the Riemann surface $\Sigma$ can be easily given in terms of the original immersion $\phi$, and the fact that it vanishes can be geometrically interpreted. For that, notice that, according to the definition (14),

$$
\alpha=\left\langle\nabla_{\frac{\partial}{\partial z}} \nabla_{\frac{\partial}{\partial z}} R, \nabla_{\frac{\partial}{\partial z}} \bar{R}\right\rangle \otimes(d z)^{3} .
$$

Now, from (8) and (2), one can see that, for each $\xi \in N$,

$$
\begin{gathered}
\left(\nabla_{\frac{\partial}{\partial z}} \nabla_{\frac{\partial}{\partial z}} R\right)(\xi)=\left\langle\nabla_{\frac{\partial}{\partial z}}^{\perp} \nabla_{\frac{\partial}{\partial z}}^{\perp} H, \xi\right\rangle(A+\phi)+\left\langle\nabla_{\frac{\partial}{\partial z}}^{\perp} H, \xi\right\rangle \phi_{z} \\
-\frac{\partial \log \left|\phi_{z}\right|^{2}}{\left|\phi_{z}\right|^{2}}\left\langle\sigma_{z z}, \xi\right\rangle \phi_{\bar{z}}-\frac{1}{\left|\phi_{z}\right|^{2}}\left\langle\nabla_{\frac{\partial}{\partial z}}^{\perp} \sigma_{z z}, \xi\right\rangle \phi_{\bar{z}}+\left\langle\sigma_{z z}, \xi\right\rangle(\phi-H) .
\end{gathered}
$$

So, we have

$$
\left\langle\nabla_{\frac{\partial}{\partial z}} \nabla_{\frac{\partial}{\partial z}} R, \nabla_{\frac{\partial}{\partial z}} \bar{R}\right\rangle(\xi, \bar{\xi})=\left\langle\sigma_{z z}, \xi\right\rangle\left\langle\nabla_{\frac{\partial}{\partial z}}^{\perp} H, \bar{\xi}\right\rangle-\left\langle\sigma_{z z}, \bar{\xi}\right\rangle\left\langle\nabla_{\frac{\partial}{\partial z}}^{\perp} H, \xi\right\rangle .
$$

Therefore, the expression of $\alpha$ in terms of $\phi$ is

$$
\alpha=\left\langle\nabla_{\frac{\partial}{\partial z}}^{\perp} H, J \sigma_{z z}\right\rangle(d z)^{3} .
$$

So, the 3-differential $\alpha$ vanishes on $\Sigma$ when

$$
\nabla_{\frac{\partial}{\partial z}}^{\perp} H \quad \text { and } \quad \sigma_{z z} \text { are proportional, }
$$

and this is, from (8), equivalent to either of the two facts

$$
\begin{array}{llll}
\partial R & \text { and } & \partial \bar{R} & \text { are proportional, } \\
\bar{\partial} R & \text { and } & \bar{\partial} R & \text { are proportional. }
\end{array}
$$

Now we shall state and prove the first main result in this paper, where we shall see that, in the genus zero case, the conformal Gauss map of a Willmore immersion into the four-sphere must be holomorphic, or be antiholomorphic, or reduce its codimension.

Theorem 4. Let $\phi: \mathbb{P}^{1} \rightarrow \mathbb{S}^{4}$ be a conformal immersion which is critical for the Willmore functional, and let $\mathcal{G}: \mathbb{P}^{1} \rightarrow \mathbb{Q}_{1}^{4} \subset \mathbb{P}_{1}^{5}$ be its conformal Gauss map. Then $\mathcal{G}$ is holomorphic, or $\mathcal{G}$ is antiholomorphic, or the image of $\mathcal{G}$ lies in a null totally geodesic complex hypersurface of the indefinite hyperquadric $\mathbb{Q}_{1}^{4}$.

Proof. Let us exclude the umbilical case (see Remark 4) where $\sigma_{z z}$ is identically zero, and so $\mathcal{G}$ is constant and work on the open dense set $\Omega \subset \mathbb{P}^{1}$ of the nonumbilical points of $\phi$. Then $\mathcal{G}: \Omega \rightarrow \mathbb{Q}_{1}^{4}$ is a minimal immersion. Hence, since $\mathbb{Q}_{1}^{4}$ is a Kähler manifold, those points of $\Omega$ where $\mathcal{G}$ is either holomorphic or antiholomorphic are isolated, provided that $\mathcal{G}$ itself is neither holomorphic nor antiholomorphic. Then, let us also exclude the cases where the conformal Gauss map is either holomorphic or antiholomorphic. As a consequence, from (10) and comments after it, we may assume that $\partial R$ and $\bar{\partial} R$ do not vanish simultaneously at the points of $\Omega$. Thus

$$
V=\operatorname{span}\{R, \bar{R}, \partial R, \overline{\partial R}, \partial \bar{R}, \bar{\partial} R\}
$$

provides, at each point of $\Omega$, a 4-dimensional vector subspace of $\mathbb{C}_{1}^{6}$ (recall Remark 5 ) which is conjugation invariant. Consider now the map $M: \mathbb{P}^{1} \rightarrow \mathbb{C}_{1}^{6}$ given by $M=A+\phi$, which can be thought of as a nowhere vanishing null section of the 
trivial bundle $\mathbb{C}_{1}^{6}$. Then, using (5), we see that $M$ is orthogonal to $V$ at each point of $\mathbb{P}^{1}$. Thus, there exists a unique differentiable section $P \in \Gamma\left(\underline{\mathbb{C}_{1}^{6}}\right)$ such that

$$
P=\bar{P}, \quad\langle P, P\rangle=0, \quad\langle P, M\rangle=1, \quad P \perp V .
$$

Now, let us denote by $\Omega^{\prime}$ the set $\Omega$ after taking away the isolated points where $\mathcal{G}$ is holomorphic. At the points of $\Omega^{\prime}$ we have that

$$
\{R, \bar{R}, \partial R, \overline{\partial R}, M, P\}
$$

give a basis of the complex vector space $\mathbb{C}_{1}^{6}$. Therefore $\partial^{2} R$ must be expressed as a linear combination of those six sections, whose coefficients will be necessarily sections of suitable holomorphic line bundles on $\Omega^{\prime}$. Taking into account (15) and the fact that $\left\langle\partial^{2} R, \partial R\right\rangle=0$, because of the first equality in (10), and the analogous properties for $\bar{R}$, we may write

$$
\begin{gathered}
\partial^{2} R=a \otimes \partial R+b \otimes M+c \otimes P, \\
\partial^{2} \bar{R}=a^{\prime} \otimes \partial \bar{R}+b^{\prime} \otimes M+c^{\prime} \otimes P,
\end{gathered}
$$

where $a, a^{\prime} \in \Gamma\left(\kappa \mid \Omega^{\prime}\right), b, c \in \Gamma\left(\kappa^{2} \otimes N_{\mid \Omega^{\prime}}^{*}\right)$ and $b^{\prime}, c^{\prime} \in \Gamma\left(\kappa^{2} \otimes \bar{N}_{\mid \Omega^{\prime}}^{*}\right)$. Then, the 4-differential $\beta$ and the 8-differential $\gamma$ defined in (14) take on $\Omega^{\prime}$ the following form:

$$
\beta_{\mid \Omega^{\prime}}=b \otimes c^{\prime}+b^{\prime} \otimes c, \quad \gamma_{\mid \Omega^{\prime}}=4 b \otimes c \otimes b^{\prime} \otimes c^{\prime} .
$$

But, as our surface has genus zero, Proposition 3 says that

$$
b \otimes c \otimes b^{\prime} \otimes c^{\prime}=0, \quad b \otimes c^{\prime}+b^{\prime} \otimes c=0
$$

on $\Omega^{\prime}$. Now, notice that, from (20) and (21), we can deduce that, for any $\xi \in N$,

$$
\begin{aligned}
& c(\xi)=\left\langle\left(\partial^{2} R\right)(\xi), M\right\rangle=\left\langle\sigma_{z z}, \xi\right\rangle(d z)^{2} \quad \text { does not vanish on } \Omega^{\prime}, \\
& c^{\prime}(\bar{\xi})=\left\langle\left(\partial^{2} \bar{R}\right)(\bar{\xi}), M\right\rangle=\left\langle\sigma_{z z}, \bar{\xi}\right\rangle(d z)^{2} \quad \text { does not vanish on } \Omega .^{\prime}
\end{aligned}
$$

As a consequence from all that we have finally that

$$
b=\left\langle\partial^{2} R, P\right\rangle=0, \quad b^{\prime}=\left\langle\partial^{2} \bar{R}, P\right\rangle=0
$$

at the points of $\Omega^{\prime}$. These two equations are equivalent, in view of (21), to

$$
\langle\partial P, \partial R\rangle=0, \quad\langle\partial P, \partial \bar{R}\rangle=0 .
$$

But, this same $(21)$ convinces us that

$$
\langle\partial P, R\rangle=-\langle P, \partial R\rangle=0, \quad\langle\partial P, \bar{R}\rangle=-\langle P, \partial \bar{R}\rangle=0 .
$$

Moreover, it is obvious that $\langle\partial P, P\rangle=0$, because $P$ is null. Hence

$$
\partial P \text { and } P \text { are proportional on } \Omega \text {. }
$$

So, there is a 1-differential $\omega$ on the open set $\Omega$ such that

$$
\partial P=P_{z} d z=\omega P \text {. }
$$

This $\omega$ satisfies $\bar{\partial} \omega=\partial \bar{\omega}$ because $\bar{P}=P$. Since this is the integrability condition of the first order equation

$$
\partial f+f \omega=0 \quad \text { with } f: \Omega \rightarrow \mathbb{R}
$$

we obtain a (locally defined) section $Q=f P \in \Gamma\left(\underline{\mathbb{R}_{1}^{6}}\right)$ orthogonal to $V$ and with $\partial Q=0$. Therefore, we have got (locally) a non-zero null $Q \in \mathbb{R}_{1}^{6}$ such that, among other things, $\langle R, Q\rangle=0$. As $\Omega$ was dense in $\mathbb{P}^{1}$ and the conformal Gauss map $\mathcal{G}$ was analytic, we may easily conclude that, in fact, this occurs on the whole of $\mathbb{P}^{1}$ 
for a fixed $Q \in \mathbb{R}_{1}^{6}$. This and the definition (7) imply that $\mathcal{G}$ maps the Riemann sphere $\mathbb{P}^{1}$ onto the intersection of the hyperquadric $\mathbb{Q}_{1}^{4}$ with a complex hyperplane of $\mathbb{P}_{1}^{5}$ whose normal directions are null.

Remark 6. It is convenient to point out that the families of genus zero Willmore surfaces in $\mathbb{S}^{4}$ appearing in Theorem 4 above have non-empty intersection. In fact, the two first of them (with holomorphic or antiholomorphic conformal Gauss map) intersect exactly in the umbilical surfaces. The first and the third ones (holomorphic conformal Gauss map and reduction of codimension) intersect in those immersions whose conformal Gauss map is holomorphic and its image is contained in a totally geodesic null complex hypersurface of the indefinite hyperquadric $\mathbb{Q}_{1}^{4}$. In Theorem 7 below, we shall show that this is a non-trivial and interesting family.

Let us examine in a more detailed way the three classes of genus zero Willmore immersions in the four-sphere. From Lemma 1, if our Willmore immersion $\phi$ has either holomorphic or antiholomorphic conformal Gauss map, then either its corresponding twistor lifting to $\mathbb{P}^{3}$ or the twistor lifting of its antipodal immersion (which is also a Willmore immersion) is a holomorphic curve. As Friedrich [F] proved that the twistor lifting of a conformal immersion into $\mathbb{S}^{4}$, obtained, via the Penrose fibration $\phi: \mathbb{P}^{3} \rightarrow \mathbb{S}^{4}$, from a holomorphic curve $C$ in $\mathbb{P}^{3}$, coincides with $C$, we have that all the genus zero Willmore surfaces whose conformal Gauss map is either holomorphic or antiholomorphic come from rational curves in the complex projective three-space through the Penrose fibration. Suppose that $\phi$ belongs to the third class of Willmore surfaces; that is, that there is a non-zero null vector $Q \in \mathbb{R}_{1}^{6}$ such that $\langle R, Q\rangle=0$, where $R$ is the section of $N^{*} \otimes \mathbb{C}_{1}^{6}$ defined in (5). So, as a consequence, $\langle Q, \mathcal{R}\rangle=0$. Rescaling, if necessary, we may choose $Q$ to be $Q=-(A+a)$, where $a \in \mathbb{S}^{4}$. Thus, we have

$$
\langle H, \xi\rangle(1-\langle\phi, a\rangle)-\langle\xi, a\rangle=0
$$

for all $\xi \in T_{\mathbb{C}}^{\perp} \Sigma$. But this can be rewritten in the following way:

$$
H=\frac{a^{N}}{1-\langle\phi, a\rangle}=\left(\nabla^{\mathbb{S}^{4}} \log (1-\langle x, a\rangle)\right)^{N},
$$

where the upper index ${ }^{N}$ denotes the normal component with respect to $\phi$. But, this equality means that, if we put on the open set $\mathbb{S}^{4}-\{a\}$ of the four-sphere the metric $d s_{a}^{2}$ given by

$$
\left(d s_{a}^{2}\right)_{x}=\frac{1}{1-\langle x, a\rangle}\left(d s^{2}\right)_{x}, \quad x \in \mathbb{S}^{4}-\{a\},
$$

where $d s^{2}$ is the standard metric on the sphere, then the immersion $\phi: \mathbb{P}^{1}-$ $\left\{\phi^{-1}(\{a\})\right\} \rightarrow \mathbb{S}^{4}-\{a\}$ is minimal. That is, if one takes stereographic projection from the point $a \in \mathbb{S}^{4}$, one obtains a minimal surface in the Euclidean four-space. The same reasonings as in [Br1] show that this minimal surface of $\mathbb{R}^{4}$ is complete, with finite total curvature, and its ends must be embedded and planar (although all that can be deduced from direct arguments that we shall give below). All these considerations can be summarized as follows.

Theorem 5. Let $\phi: \mathbb{P}^{1} \rightarrow \mathbb{S}^{4}$ be a conformal immersion which is a critical point for the Willmore functional. Then either $\phi$ or its antipodal $-\phi$ are obtained from a rational curve in $\mathbb{P}^{3}$, via the Penrose twistor fibration, or $\phi$ is obtained from a 
complete minimal surface of $\mathbb{R}^{4}$ with finite total curvature and planar embedded ends, via the inverse of the stereographic projection.

Suppose first that $\phi: \mathbb{P}^{1} \rightarrow \mathbb{S}^{4}$ is a Willmore immersion. We have already pointed out that its regular homotopy class is completely determined by the Euler number of its normal bundle, that is, by $c(N)=2 q$, where $q \in \mathbb{Z}$ is the self-intersection number of the immersion. Changing $\phi$ to its antipodal $-\phi$ if necessary, we may assume that $c(N)=2 q \geq 0$ (in this way the second case in Theorem 5 is possible only when $\phi$ is an umbilical immersion). Then, if the twistor lifting $\mathcal{T}: \mathbb{P}^{1} \rightarrow \mathbb{P}^{3}$ of $\phi$ is holomorphic, from the equalities before (12) we have

$$
\mathcal{W}(\phi)=4 \pi \operatorname{deg} \mathcal{T}=2 \pi(1+c(N))=4 \pi(1+q) .
$$

Second, let $\phi$ be in the last case of Theorem 5 above, that is, there is $a \in \mathbb{S}^{4}$ such that $\psi: \Sigma=\mathbb{P}^{1}-\phi^{-1}(\{a\}) \rightarrow \mathbb{R}^{4}$ is a minimal immersion, where $\psi=P \circ \phi, P$ : $\mathbb{S}^{4}-\{a\} \rightarrow \mathbb{R}^{4}$ being the stereographic projection. Then, the conformal invariance of the Willmore functional (see [Br1], for example) implies that

$$
\mathcal{W}(\phi)-\int_{\mathbb{P}^{1}} K d A=-\int_{\Sigma} K_{\psi} d A_{\psi} .
$$

Thus, taking into account that the total curvature of a minimal surface in Euclidean space can be computed in terms of its Gauss map, as one can see, for instance, in HO, we have

$$
\mathcal{W}(\phi)=4 \pi+2 \pi\left(d_{1}+d_{2}\right),
$$

where $d_{1}, d_{2} \geq 0$ are the degrees of the two components of the Gauss map of $\psi$ (recall that this Gauss map goes from $\Sigma$ onto the quadric $\mathbb{Q}^{2} \cong \mathbb{P}^{1} \times \mathbb{P}^{1}$ ). On the other hand, since the integral of the normal curvature is also a conformal invariant, as Weiner remarked in [We, and the normal curvature of the minimal immersion $\psi$ can also be expressed from its Gauss map (again, see [We]), we have

$$
2 \pi c(N)=4 \pi q=\int_{\Sigma} K_{\psi}^{\perp} d A_{\psi}=2 \pi\left(d_{1}-d_{2}\right) .
$$

Therefore, finally we have obtained the following expression of the value that the Willmore functional take on $\phi$ in terms of its regular homotopy class:

$$
\mathcal{W}(\phi)=4 \pi(1+q+n)
$$

where $n=d_{2} \geq 0$. As a consequence of all these reasonings we have obtained the third main result of this paper.

Theorem 6. The critical values of the Willmore functional for genus zero immersions into the four-sphere are $4 \pi k$ with $k \in \mathbb{N}^{*}$. Moreover, in the regular homotopy class determined by the Whitney self-intersection number $q \in \mathbb{Z}$, the possible critical values are $4 \pi(1+|q|+n)$ with $n \in \mathbb{N}$, and $n=0$ if and only if the immersion (or its antipodal) comes, via the Penrose twistor fibration, from a $1+q$-degree rational curve in the complex projective space $\mathbb{P}^{3}$.

Let us introduce another invariant of the conformal immersion $\phi: \mathbb{P}^{1} \rightarrow \mathbb{S}^{4}$, which is important in the context of the Willmore functional and that was first used by Li and Yau in [LY]. Let $\mu(\phi)$ the maximum multiplicity of the points of our immersed surface. A theorem of Li and Yau [LY] and its corresponding improvement by Kusner [K2] say that

$$
\mathcal{W}(\phi) \geq 4 \pi \mu(\phi)
$$


and the equality holds if and only if there is a stereographic projection carrying $\phi$ to a minimal immersion in $\mathbb{R}^{4}$.

When $\phi$ is a Willmore immersion and does not reach the minimum value of $\mathcal{W}$ in its homotopy class, we have, from Theorem 6 , that $\mathcal{W}(\phi)=4 \pi(1+|q|+n)$ for some $n \in \mathbb{N}^{*}$, and, from Theorem 5 , that there exists a stereographic projection which changes $\phi$ into a minimal immersion in $\mathbb{R}^{4}$. So, in this case, the cited result by Li, Yau and Kusner asserts that $\mu(\phi)=1+|q|+n$.

When $\phi$ is a Willmore immersion attaining the minimum value $4 \pi(1+|q|)$ of the Willmore functional $\mathcal{W}$ in its homotopy class, we have only the inequality $\mu(\phi) \leq 1+|q|$, and equality occurs if and only if $\phi$ is obtained simultaneously from a rational curve with degree $1+|q|$ in $\mathbb{P}^{3}$, via the Penrose fibration, and from a complete minimal surface of $\mathbb{R}^{4}$ with finite total curvature and embedded planar ends. Then, from $(22)$, we deduce that $n=d_{2}=0$, and so, one of the two components of the Gauss map of that minimal surface is holomorphic. One can see in [HO that this is equivalent to the fact that the minimal surface is a holomorphic curve with respect to a certain complex structure on $\mathbb{R}^{4}$. Also, we shall be able to reflect the equality $\mu(\phi)=1+|q|$ on the behavior of the twistor lifting $\mathcal{T}$ of the immersion $\phi$. Since there is a point $a \in \mathbb{S}^{4}$ in the image of $\phi$ with multiplicity $1+|q|$, the vertical complex line $L_{a} \subset \mathbb{P}^{3}$ corresponding to $a$ intersects the image of $\mathcal{T}$ with multiplicity $1+|q|=\operatorname{deg} \mathcal{T}$. Then the complex plane of $\mathbb{P}^{3}$ determined by $L_{a}$ and a point of the image of $\mathcal{T}$ not belonging to $L_{a}$ cuts the image of $\mathcal{T}$ in $1+\operatorname{deg} \mathcal{T}$ points. Hence, the image of the twistor lifting of $\phi$ lies in a plane of the complex projective space. So, we may state the following theorem, which finally solves the problem of finding the Willmore surfaces into $\mathbb{S}^{4}$ coming from minimal surfaces into Euclidean space and holomorphic curves in the complex projective three-space (see [We] for some partial results about this same problem).

Theorem 7. Let $\phi: \mathbb{P}^{1} \rightarrow \mathbb{S}^{4}$ be a conformal immersion which attains the minimum $4 \pi(1+|q|)$ of the Willmore functional in its regular homotopy class. Then all the points of its image have multiplicity at most $1+|q|$, and the following facts are equivalent:

1. There exists a point in the image of $\phi$ with maximum multiplicity $1+|q|$.

2. The conformal Gauss map $\mathcal{G}: \mathbb{P}^{1} \rightarrow \mathbb{Q}_{1}^{4}$ of $\phi$ is holomorphic or antiholomorphic, and its image lies in a totally geodesic null complex hypersurface.

3. The image of the twistor lifting $\mathcal{T}: \mathbb{P}^{1} \rightarrow \mathbb{P}^{3}$ of $\phi$ (if $q \geq 0$, or the twistor lifting of the antipodal $-\phi$, if $q \leq 0$ ) lies in a plane $\mathbb{P}^{2} \subset \mathbb{P}^{3}$.

4. Either $\phi$ or $-\phi$ is obtained from a $1+|q|$-degree rational planar curve, via the Penrose twistor fibration.

5. $\phi$ is obtained from a genus zero complex curve in $\mathbb{C}^{2}$ with finite total curvature $-4 \pi|q|$ and planar embedded ends.

When $\phi$ is a Willmore immersion with $\mathcal{W}(\phi)=4 \pi(1+|q|+n), n \neq 0$, then the image of $\phi$ has at least one point with multiplicity $1+|q|+n$.

Notice that, as a consequence of these last theorems, we can observe that there are genus zero Willmore immersions into $\mathbb{S}^{4}$ which do not come from minimal surfaces of $\mathbb{R}^{4}$ via the stereographic projection (this could not occur when the ambient space was $\mathbb{S}^{3}$ ). They are necessarily absolute minima for $\mathcal{W}$ in their regular homotopy class, and are produced from non-planar rational curves in $\mathbb{P}^{3}$ through the Penrose twistor fibration. These curves must have degree at least three, and so the mininum value of $\mathcal{W}$ for these type of Willmore immersions is $12 \pi$. In fact, as 
the Veronese embedding (rational normal curve in $[\mathrm{GH}]$ ) from $\mathbb{P}^{1}$ in $\mathbb{P}^{3}$ is the only (up to projective transformation) possible degree three curve, the only genus zero Willmore immersion $\phi$ in $\mathbb{S}^{4}$ with $\mathcal{W}(\phi)=12 \pi$ without triple points is the so-called Veronese (minimal) immersion (and all its twistor deformations).

Now, take away this kind of genus zero Willmore surfaces which are absolute minima for $\mathcal{W}$ in its homotopy class and have not a point with maximum multiplicity. In the remaining cases, our immersion $\phi: \mathbb{P}^{1} \rightarrow \mathbb{S}^{4}$ yields a complete minimal immersion $X: \mathbb{P}^{1}-D \rightarrow \mathbb{R}^{4}$ with finite total curvature $-4 \pi(k-1)$ and embedded planar ends, where $D=\left\{p_{1}, \ldots, p_{k}\right\}$ is a finite set (the ends set) and $\mathcal{W}(\phi)=4 \pi k$. Then $\partial X$ is a meromorphic $\mathbb{C}^{4}$-valued 1-differential with simple poles at the points of $D$. Moreover, the fact that the ends of $X$ are planar is equivalent to these poles having vanishing residues (see Theorem $\mathrm{F}$ in $[\mathrm{Br} 1)$ ). So, the conjugate minimal immersion of $X$ exists; that is, $X$ is the real part of a meromorphic curve $f: \mathbb{P}^{1} \rightarrow \mathbb{C}^{4}$ whose polar divisor is $p_{1}+\cdots+p_{k}$. As $X$ is a conformal immersion, the curve $f$ must be an immersion with null tangents. Now one can repeat the arguments in [Br2] and consider the non-degenerate hyperquadric $\mathbb{Q}^{4}$ given by

$$
2 z_{0} z_{5}-\left(z_{1}\right)^{2}-\left(z_{2}\right)^{2}-\left(z_{3}\right)^{2}-\left(z_{4}\right)^{2}=0
$$

in the complex projective space $\mathbb{P}^{5}$ as the conformal compactification of the affine space $\mathbb{C}^{4}$, by using the embedding

$$
\left(z_{1}, z_{2}, z_{3}, z_{4}\right) \in \mathbb{C}^{4} \longmapsto\left[2,2 z_{1}, 2 z_{2}, 2 z_{2}, 2 z_{3}, 2 z_{4},\left(z_{1}\right)^{2}+\left(z_{2}\right)^{2}+\left(z_{3}\right)^{2}+\left(z_{4}\right)^{2}\right] \in \mathbb{Q}^{4} .
$$

The composition of the curve $f$ with this embedding provides us a holomorphic null immersion $F: \mathbb{P}^{1} \rightarrow \mathbb{Q}^{4} \subset \mathbb{P}^{5}$ with degree $k$. Therefore, when $\mathcal{W}(\phi) \leq 16 \pi$, we have $k=\operatorname{deg} F \leq 4$, and the image of $F$ lies in a hyperplane $H$ of $\mathbb{P}^{5}$ (see GH] ). But the intersection $\mathbb{Q}^{4} \cap H$ is a hyperquadric in $H$. If this hyperquadric is non-degenerate, then we can assume that the image of $F$ lies in $\mathbb{Q}^{3} \subset \mathbb{P}^{4}$, and so the minimal immersion $X$ has image into $\mathbb{R}^{3}$ and, finally, $\phi\left(\mathbb{P}^{1}\right)$ is contained in an umbilical three-sphere of $\mathbb{S}^{4}$. In this case, we may apply the results in [Br2] and conclude that the only possible values of $\mathcal{W}(\phi)$ are $4 \pi$, when $\phi$ is umbilical, and $16 \pi$, and, in this case, $\phi$ is produced essentially from a genus zero minimal surface with four embedded planar ends and tetrahedral symmetry which has appeared in several contexts (see $\mathrm{Br} 1, \overline{\mathrm{RT}}$ ] and $[\mathrm{MRO}$ ). On the other hand, assume now that the intersection $\mathbb{Q}^{4} \cap H$ gives a degenerate hyperquadric in $H$. Then, choosing suitable homogeneous coordinates $\left[z_{0}, \ldots, z_{5}\right]$ on $\mathbb{P}^{5}$, this intersection can be written in this way:

$$
z_{0}=0, \quad\left(z_{1}\right)^{2}+\left(z_{2}\right)^{2}+\left(z_{3}\right)^{2}+\left(z_{4}\right)^{2}=0 .
$$

Now, it is not difficult to check that the null curves in that intersection are of the form $\left[z_{0}(t), z_{1}(t), \ldots, z_{4}(t)\right]$, where the projected curve $\left[z_{1}(t), \ldots, z_{4}(t)\right]$ is a null curve in the conic $\mathbb{Q}^{2} \subset \mathbb{P}^{3}$. Therefore, the image of such a null curve in the intersection must lie in a plane of $\mathbb{P}^{5}$. As a consequence, $k=\operatorname{deg} F \leq 2$. Then the total curvature of the minimal immersion $X$ is zero or $-4 \pi$. In the first case, $X$ determines a plane and $\phi$ is umbilical; and, in the second case, $X$ is a complete minimal surface in $\mathbb{R}^{4}$ with total curvature $-4 \pi$. All these minimal immersions were found in [HO] and are the complex graphs

$$
z \in \overline{\mathbb{C}} \longmapsto\left(z, a z+\frac{1}{z}\right) \in \mathbb{C}^{2} \equiv \mathbb{R}^{4},
$$


with $a \in \mathbb{R}$. Taking into account Theorem 7 , our immersion $\phi$ can be obtained from some of these minimal surfaces, via inversions, or from a degree 2 holomorphic planar curve in some $\mathbb{P}^{2}$ of $\mathbb{P}^{3}$, that is, from a degree 2 Veronese embedding. All the $\phi$ obtained in this way have exactly one double point and are regularly homotopic to the Whitney sphere described in [Wei] (see also CU] for other interesting characterizations). In this way, we can classify all the genus zero Willmore surfaces of the four-sphere with a small value of $\mathcal{W}$ and show that the forbidden values $8 \pi$ and $12 \pi$ when the ambient space was three-dimensional occur in our case.

Corollary 8. Let $\phi: \mathbb{P}^{1} \rightarrow \mathbb{S}^{4}$ be a Willmore immersion such that $\mathcal{W}(\phi) \leq 12 \pi$. Then, one of the following alternatives occurs:

1. $\mathcal{W}(\phi)=4 \pi$ and $\phi$ is umbilical.

2. $\mathcal{W}(\phi)=8 \pi$ and $\pm \phi=P \circ V_{2}$, where $P: \mathbb{P}^{3} \rightarrow \mathbb{S}^{4}$ is the Penrose twistor fibration and $V_{2}: \mathbb{P}^{1} \rightarrow \mathbb{P}^{2} \subset \mathbb{P}^{3}$ is the degree two Veronese embedding. In this case $\phi$ has exactly one double point and $q= \pm 1$ (when $q=1$ it is homotopic to the Whitney sphere).

3. $\mathcal{W}(\phi)=12 \pi$ and $\pm \phi=P \circ V_{3}$, where $V_{3}: \mathbb{P}^{1} \rightarrow \mathbb{P}^{3}$ is the degree three Veronese embedding. In this case $q= \pm 2$, and $\phi$ is a twistor deformation of the Veronese minimal embedding from $\mathbb{S}^{2}$ into $\mathbb{S}^{4}$.

4. $\mathcal{W}(\phi)=12 \pi$ and $\phi$ is obtained, via the inverse of the stereographic projection, from some of the complete minimal surfaces in $\mathbb{R}^{3}$ (so $q=0$ in this case) with four embedded planar ends (pointing to the vertices of a tetrahedron) of Bryant-Rosenberg-Toubiana.

\section{REFERENCES}

[Bl] W. Blaschke, Vorlesungen Uber Differentialgeometrie III, Springer, Berlin, 1929.

[Br1] R.L. Bryant, A duality theorem for Willmore surfaces, J. of Diff. Geom., 20(1984), 23-53. MR 86j:58029

[Br2] R.L. Bryant, Surfaces in conformal geometry, Proc. of Symp. in Pure Math., 48(1988), 227-240. MR 89m:53102

[CU] I. Castro, F. Urbano, Lagrangian surfaces in the complex Euclidean plane with conformal Maslov form, Tôhoku Math. J., 45(1993), 565-582. MR 94j:53064

[E] N. Ejiri, Willmore surfaces with a duality in $S^{N}(1)$, Proc. London Math. Soc., 57(1988), 383-416. MR 89h:53117

[ES] J. Eells, S. Salamon, Twistorial constructions of harmonic maps of surfaces into fourmanifolds, Ann. Scuola Norm. Sup. Pisa, 12(1985), 589-640. MR 87i:58042

[F] T. Friedrich, On surfaces in four-spaces, Ann. Global Anal. Geom., 2(1984), 257-287. MR 86h:53061

[GH] P. Griffiths, J. Harris, Principles of Algebraic Geometry, Wiley-Interscience, New York, 1978. MR 80b:14001

[Hi] M.W. Hirsch, Immersions of manifolds, Trans. Amer. Math. Soc., 93(1959), 242-276. MR 22:9980

[HO] D. Hoffman, R. Osserman, The geometry of the generalized Gauss map, Mem. Amer. Math. Soc., 236(1980). MR 82b:53012

[KM] J.L. Koszul, B. Malgrange, Sur certaines structures fibres complexes, Arch. Math., 9(1958), 102-109. MR 24:A1729

[K1] R. Kusner, Conformal geometry and complete minimal surfaces, Bull. Amer. Math. Soc., 17(1987), 291-295. MR 88j:53008

[K2] R. Kusner, Comparison surfaces for the Willmore problem, Pacific Math. J., 138(1989), 317-345. MR 90e:53013

[KS] R. Kusner, N. Schmitt, The spinor representation of surfaces in space, preprint.

[LS] R. Lashof, S. Smale, On immersions of manifolds in Euclidean space, Ann. of Math., 68(1958), 562-583. MR 21:2246 
[LY] P. Li, S.T. Yau, A new conformal invariant and its applications to the Willmore conjecture and first eigenvalue of compact surfaces, Invent. Math., 69(1982), 269-291. MR 84f:53049

[MR] S. Montiel, A. Romero, Complex Einstein hypersurfaces of indefinite complex space forms, Math. Proc. Cambridge Phil. Soc., 93(1983), 495-508. MR 85f:53049

[MRo] S. Montiel, A. Ros, Minimal immersions of surfaces by the first eigenfunctions and conformal area, Invent. Math., 83(1986), 153-166. MR 87d:53109

[R] A. Ros, The Willmore conjecture in the real projective space, Math. Res. Lett. 6 (1999), 487-493.

[RT] H. Rosenberg, E. Toubiana, Some remarks on deformations of minimal surfaces, Trans. Amer. Math. Soc., 295(1986), 491-499. MR 88a:53005b

[S] S. Salamon, Topics in four-dimensional Riemannian geometry, Lecture Notes in Mathematics, 1022(1982), 34-124. MR 85i:53002

[We] J.L. Weiner, On an inequality of P. Wintgen for the integral of the square of the mean curvature, J. London Math. Soc., 34(1986), 148-158. MR 87k:53147

[Wei] A. Weinstein, Lectures on symplectic manifolds, Conf. Board Math. Sci., Regional Conf. Ser. Math., vol. 29, Amer. Math. Soc., Providence, RI, 1977. MR 57:4244

[Wi1] T.J. Willmore, Note on embedded surfaces, An. Sti. Univ. "Al. I. Cuza" Iasi Sect. I a Mat., 11(1965), 493-496. MR 34:1940

[Wi2] T.J. Willmore, Mean curvature of Riemannian immersions, J. London Math. Soc., 3(1971), 307-310. MR 44:959

[Win] P. Wintgen, On the total curvature of surfaces in $\mathbb{E}^{4}$, Colloq. Math., 39(1978), 289-296. MR 80f:53037

[Wo] J.A. Wolf, Spaces of Constant Curvature, McGraw-Hill, New York, 1967. MR 36:829

Departamento de Geometría y Topología, Universidad de Granada, E-18071 Granada, SPAIN

E-mail address: smontiel@goliat.ugr.es 